\title{
Un riconoscimento ufficiale (SCOPUS): Scienze Regionali è una rivista internazionale
}

\author{
Roberta Capello \\ Direttore Scienze Regionali
}

Dopo dieci anni dalla sua costituzione, Scienze Regionali ha ottenuto il riconoscimento SCOPUS. Non è un risultato da poco, e per questo motivo vorrei condividere con voi i vantaggi e le sfide di questo obiettivo raggiunto.

Scienze Regionali è solo la terza rivista italiana nelle Scienze Sociali a essere riconosciuta tra le riviste SCOPUS, a segnalare la complessità di questa operazione, la competizione che esiste a livello internazionale per essere riconosciuti tra le riviste "quotate", e non ultimo il lavoro scientifico e di redazione che è richiesto per raggiungere tale riconoscimento.

Per quanto ci riguarda, posso dire che il riconoscimento è il risultato di un lavoro di squadra dell'intero comitato di redazione, che con professionalità, passione e dedizione al "progetto culturale" ha lavorato perché la rivista raggiungesse i livelli internazionali, impegnandosi nell'attrarre articoli da studiosi stranieri noti, lanciando numeri speciali su temi di frontiera, sostenendo note e dibattiti firmate da studiosi internazionali, imponendo seri referaggi e revisioni spesso radicali agli autori, in linea con le riviste internazionali, e producendo una scelta di recensioni sulle ultime monografie internazionali pubblicate. Il lavoro ha dato i frutti sperati, e ha aperto speranze per il secondo importante riconoscimento a cui già da tempo lavoriamo, quello ISI.

Scienze Regionali è ormai riconosciuta a tutti gli effetti una rivista internazionale. Che cosa comporta tutto questo e come si presenta il nostro futuro?

Senz'altro esistono vantaggi dalla posizione raggiunta. Scienze Regionali diventa un canale editoriale attraverso il quale far conoscere alla comunità scientifica internazionale nostri idee, contributi, metodi e strumenti: abbiamo pertanto una possibilità in più rispetto al passato di far conoscere la cultura italiana nelle Scienze Regionali. Al tempo stesso, diventa sempre più un canale di pubblicazione per incanalare in Italia le nuove idee e i nuovi strumenti sviluppati a livello internazionale.

Il nostro futuro è roseo, anche se non privo di sfide. L'obiettivo raggiunto ci impone con ancor più forza di mantenerci al passo con gli standard delle riviste internazionali, vigilando sulla qualità dei contributi, dei referaggi e del lavoro editoriale. Entriamo nella competizione tra riviste internazionali per ottenere gli articoli più innovativi, e al tempo stesso a livello nazionale sentiamo la necessità di catturare le principali idee che nascono, perché la cultura italiana nel campo delle Scienze Regionali sia ben rappresentata.

Tutto questo non è un lavoro da poco. Se il comitato di redazione, nelle persone di Roberto Basile, Ugo Fratesi, Stefano Moroni, Marco Percoco e Corrado Zoppi, mi ha aiutata negli ultimi tre anni per ottenere il riconoscimento SCOPUS (lascia- 
temi esprimere tutto il mio sincero riconoscimento a questi colleghi), il direttivo AISRe ha nominato un nuovo comitato di redazione, in carica dal 1 Luglio - composto da Giulio Cainelli, Edoardo Marcucci, Ezio Micelli, Stefano Moroni, Laura Resmini (ai quali do il benvenuto) - con il quale affronterò le sfide che ho sopra menzionato, attraverso un nuovo progetto editoriale, che dia un ulteriore slancio alla nostra rivista.

Tuttavia, perché tutti i nostri obiettivi si realizzino, serve la partecipazione di tutta la comunità scientifica, di tutti voi. Senza il vostro contributo la rivista non può esistere: un contributo come autori, pronti a giocare con le regole del gioco internazionale - competitive ma anche spesso fastidiose per la puntigliosità che impongono - e un contributo come lettori attenti, interessati e perché no, critici.

Spero sinceramente che il riconoscimento ottenuto serva a tutta la comunità scientifica italiana perché la qualità dei suoi lavori venga riconosciuta a livello internazionale e, non ultimo, ai nostri giovani, ai quali il mondo accademico ormai impone di scegliere i loro canali di pubblicazione esclusivamente tra le riviste indicizzate.

Con la speranza che tutto lo sforzo richiesto nel realizzare la rivista serva a tutti noi, spero di ricevere presto vostri importanti contributi. 